\title{
Erratum to: Existence and concentration of positive solutions for semilinear Schrödinger-Poisson systems in $\mathbb{R}^{3}$
}

\author{
Jun Wang · Lixin Tian · Junxiang Xu · Fubao Zhang
}

Published online: 6 November 2012

(C) Springer-Verlag Berlin Heidelberg 2012

\section{Erratum to: Calc. Var. DOI 10.1007/s00526-012-0548-6}

In the previous paper [1], we proved the existence and concentration of positive ground state solution for Schrödinger-Poisson systems. In Sect. 1 we introduced the conditions $\left(\mathcal{B}_{0}\right)-\left(\mathcal{B}_{1}\right)$ and the sets

$$
\mathcal{K}_{1}=\left\{x \in \mathscr{A}: b(x)=b\left(x_{a}\right)\right\} \cup\left\{x \notin \mathscr{A}: b(x)>b\left(x_{a}\right)\right\}
$$

and

$$
\mathcal{K}_{2}=\left\{x \in \mathscr{B}: a(x)=a\left(x_{b}\right)\right\} \cup\left\{x \notin \mathscr{B}: a(x)<a\left(x_{a}\right)\right\} .
$$

The technique of introducing the conditions $\left(\mathcal{B}_{0}\right)$ - $\left(\mathcal{B}_{1}\right)$ and the sets $\mathcal{K}_{1}$ and $\mathcal{K}_{2}$ is very important for the proof. However, we missed this fact: the idea of introducing these conditions and sets is actually due to Professor Ding, who first reported it in several academic conferences in 2010, and then in [2,3] Ding and Liu explored the idea to develop an argument to study the existence and concentration of semiclassical solutions for Dirac systems and Schrodinger equations with magnetic fields. So here we point out that the construction of the conditions $\left(\mathcal{B}_{0}\right)-\left(\mathcal{B}_{1}\right)$ and the sets $\mathcal{K}_{1}$ and $\mathcal{K}_{2}$ in [1] is actually motivated by Ding and Liu's idea in [2,3].

The online version of the original article can be found under doi:10.1007/s00526-012-0548-6.

J. Wang $(\varangle) \cdot$ L. Tian

Department of Mathematics, Jiangsu University, Zhenjiang 212013, Jiangsu, People's Republic of China e-mail: wangmath2011@126.com

L. Tian

e-mail: tianlx@ujs.edu.cn

J. Xu $\cdot$ F. Zhang

Department of Mathematics, Southeast University, Nanjing 210096, People's Republic of China e-mail: xujun@seu.edu.cn

F. Zhang

e-mail: zhangfubao@seu.edu.cn 


\section{References}

1. Wang, J., Tian, L., Xu, J., Zhang, F.: Existence and concentration of positive solutions for semilinear Schrödinger-Poisson systems in $\mathbb{R}^{3}$. Calc. Var. doi:10.1007/s00526-012-0548-6

2. Ding, Y., Liu, X.: Semi-classical limits of ground states of a nonlinear Dirac equation. J. Differ. Equ. 252, 4962-4987 (2012)

3. Ding, Y., Liu, X.: Semiclassical solutions of Schrödinger equations with magnetic fields and critical nonlinearities. Manuscripta Math. (2012). doi:10.1007/s00229-011-0530-1 